EQUIIIBRITM SITUATIONS IN GAMES WITH A HIERARCHICAL STRUCTURE OF THE VECTOR OF CRITERTA

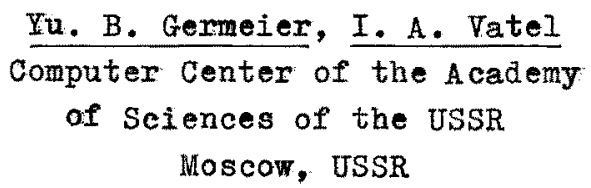

Among models of decision making in n-person games with vector criteria it is possible to distinguish one class of models in which the vector of criteria have a so-called hierarchical structure. Multi-level economic and social systems are used as the basis of such models. Each of these "large" systems are sub-divided into a series of groups, which in their turn are again sub-divided, etc., until some elementary unit remains - a player, constituting the "subject" for making a decision.

It is possible to attribute to each group as a unitary system the propensity to increase certain criteria, and generally speaking, their magnitude depends on the actions of all the members of the given group. At the same time there exists a personal (individual) interest of the given player, whose magnitude, an idealization to a known degree, depends only upon his own actions. These actions for increasing social and personal criteria may be interpreted as a distribution by the player of his resources among the interests of the group, of which he is a member at various levels.

In practice there are many examples of models of such type. Thus, in models of the production sphere three categories of interests may be distinguished - a public interest, the interest of the production unit collective (enterprise, collective farm), and a personal material interest of a hard-working person. In sociological models a system of the interests of an individual, family or social group may be considered. Decision making in the sphere of international relations is connected with an analysis of national, regional and general world interests. If we limit ourselves to the study of models in which the choice of strategy by the players is carried out on a voluntary basis (as, for example, in models of international cooperation pertaining to the protection of the environment), then equilibrium situations may serve as one of the rational principles of decision making. Such models are considered below. 


\section{A description of the model.}

Let $n$ players establish a $(m+1)$-leveled society $(k=\{k: k=1, \ldots, m\} \quad$ is the set of index numbers of the levels), that are sub-divided into a series of hierarchically organized groups, the society itself - a zero-level group is determined by the set of the numbers of all the players $s^{\circ}=\{i: i=1, \ldots, n\}$. The society is sub-divided into groups of the first level, each of which in its turn, into groups of the second level, etc. In this way, each group $j$ of the $k-t h$ level may be described by the set $f_{j}$ of the number of players that compose it, whereupon the index assumes the value from the set of the numbers of the group, that exist at a given level $k$. At the final $m$-th level each group consists of one player, i.e. $\rho_{i} n=\{i\}$

A criterion (interest) $w_{j}^{k}$ is associated with each group, which it strives to increase. Iet us assume, that the magnitude of this criterion depends on the values $x_{y}$, that are chosen by only the players of whom it is composed, i.e.

$$
w_{j}^{k}=f_{j}^{k}\left(x_{s} \mid s \in j_{j}^{k}\right)
$$

Therefore each player $i$ has a $k(m+1)$-dimensional vector of interests with component; $w_{j}$ ( in addition the numbers of the groups are such that $\left.\xi_{j}^{k} \supset i, k \in K\right)$. Each player is regarded to have a propensity to increase the compromise criteria, composed

of $(1), i . e$.

$$
w_{i}=w_{i}\left(w_{j}^{k} \mid s_{j}^{k} \supset i, k \in k\right) .
$$

It is assumed that the $i-t h$ player possesses his own vectorial resource $a_{i}$, that he allocates anong the interests of the groups at all levels of which he is a member, i.e.,

$$
\begin{aligned}
x_{i}=\left\{x_{i}^{0}, \ldots, x_{i}^{m}\right\} & \\
& \sum_{k=0}^{m} x_{i}^{k}=a_{i}, x_{i}^{k} \geqslant 0, k \in K
\end{aligned}
$$

and

Iet us adopt the compromise criteria (2), according to $[1]$, in the form

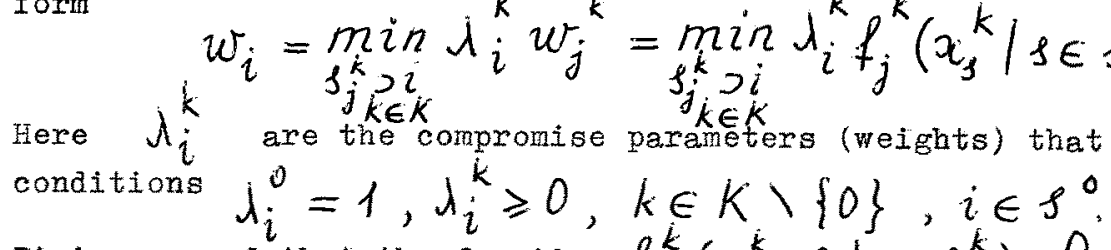

It is assumed that the function $f_{j}^{k}\left(x_{j}^{k}=0 \mid s \in s_{j}^{k}\right)=0$ increases monotonically with the growth of any one of the components of the 
vector $x_{3}{ }^{k}$ and

$$
f_{j}^{k}\left(x_{s}^{k}=0 \mid s \in s_{j}^{k}\right)=0 .
$$

\section{Equilibrium situations .}

One of the widely utilized principles for a joint decision making in n-person games is derived from equilibrium situations [2] . As we shall see below, they possess a series of important properties, in the given model, that speak in favour of their utilization.

Above all. we shall be interested in the existence of equilibrium situations in pure strategies. Here the following theorem is relevant [3].

Theorem 1. In a game with scalar resources $a_{i}$, in order that the situation $\bar{x}=\left(\bar{x}_{1}, \ldots, \bar{x}_{n}\right)$ be an equilibrium situation, it is necessary and sufficient, that there exist such an and $K_{i}^{\prime \prime}$ af partitions of the set $K \backslash\{n\}$ into two subsets $K_{i}$

$$
\left(K_{i}^{\prime} \cup K_{i}^{\prime \prime}=K \backslash\{m\}, K_{i}^{\prime} \cap K_{i}^{\prime \prime}=\varnothing\right) \text {, that vector }
$$

the following system for all $i \in \rho^{\circ}$ :

$$
\begin{aligned}
& \lambda_{i}^{m} f_{i}^{m}\left(\bar{x}_{i}^{m}\right)=\lambda_{i}^{k} f_{j}^{k}\left(\bar{x}_{s}^{k} \mid s \in s_{j}^{k}\right), \bar{x}_{i}^{k} \geqslant 0, k \in K_{i}^{\prime}, \\
& \lambda_{i}^{m} f_{i}^{m}\left(x_{i}^{m}\right)<\lambda_{i}^{k} f_{j}^{k}\left(\bar{x}_{j}^{k} \mid s \in s_{j}^{k}\right), \bar{x}_{i}^{k}=0, k \in K_{i}^{\prime \prime}
\end{aligned}
$$

where for all $k \in K \backslash\{m\}$ the indicies $j$ are determined by the conditions

$$
\rho_{j}^{k} \supset i
$$

If the $\alpha_{i}$ 's form a vector, then (6) turn out to be the only necessary conditions for an equilibrium situation. Let us note that in order to prove theorem $1 f_{i}^{m}(0)=0, i \in j^{D}$ are the only sufficient conditions; this is also true of $f_{j}^{k}\left(x_{j}^{k}=0 \mid s \in s_{j}^{k}\right)>0, k \in K \backslash\{m\}$.

This fact has a significance for dynamic models where from step to step an accumulation of the values of "social" criteria may take place. It should be emphasized as well that the conditions

$$
f_{i}^{m}(0)=0, i \in \rho^{0} \text { lead to a compulsory setting aside of }
$$
means for personal criteria, i.e., $\bar{x}_{i}^{m}>0, i \in s^{0}$ and it follows from $(6)$ that in any equilibrium situation, player

$i$ measures his resuIts by "a most individual measure" $d_{i}^{m} f_{i}^{m}\left(\bar{x}_{i}^{m}\right)$. 
Theorem 1 reduces the problem of searching for an equilibrium situation to the solution of a system composed of $n$ systems of equations of the type (3), (6). In this event it is necessary to carefully examine $2^{\mathrm{mn}}$ such systems in order to search for all equilibrium situations.

An important class of societies, for which the analysis is substantially simplified, are the hierarchically coordinated societies, given by the conditions

$$
\lambda_{i}^{k}=d_{j}^{k}, i \in s_{j}^{k}, k \in K_{3}
$$

i.e. all players of group $j$ of the $k$-th level attach similar weights to the criteria of this group. As has been shown in $[3]$, in an equilibrium situation under conditions (7) for any player $i$ a number $\tau_{i}$ may be found such that

$$
K_{i}^{\prime}=\left\{\gamma_{i}, \ldots, m-1\right\}, K_{i}^{\prime \prime}=\left\{0,1, \ldots, \tau_{i}-1\right\},
$$

In other words, a non-allocation of means, above all, affects the criteria of rather high levels.

A society is described as harmonious if $K_{i}^{\prime \prime}=\phi_{\text {for a.I }}$ $i \in \rho^{0}$ in any equilibrium situation, $i . e$. generally speaking the players distribute their resources among the interests of all levels. It is not difficult to show that a harmonious society is bierarchically coordinated. In this society there exists one equilibrium situation (or several that are equivalent) that is best. Thus, in a harmonious society the interests of all the players, in the sense of choosing an equilibrium situation, coincide. It should be emphasized here that in the case of a non-uniqueness of this best situation, the players should trade information in order to arrive at a joint coordinated choice.

Let us note that if the society is not hierarchically coordinated, then equilibrium situations in pure strategies may not exist.

\section{A two-level society.}

The situation $m=1$ is a most simple model for the analysis of the problem of the combination of "personal and social interests". Let us write the compromise criteria (2) in the form

$$
w_{i}=\min \left\{f^{\circ}\left(x_{1}, \ldots, x_{n}\right), d_{i} f_{i}\left(a_{i}-x_{i}\right)\right\} \text {. }
$$

For purposes of simplification, here the upper indices are omitted. Let us note that conditions of hierarchical co-ordination (7) are fulfilled. 
Without disturbing the community, let us assume that the number of players in the society are ordered in the following mannex

$$
\lambda_{1} f_{1}\left(a_{1}\right) \geqslant \lambda_{2} f_{2}\left(a_{2}\right) \geqslant \ldots \geqslant \lambda_{n} f_{n}\left(a_{n}\right)
$$

Then the following theorem is true.

Theorem 2. In a two-level society an equilibrium situation $\left(\bar{x}_{1}, \ldots, \bar{x}_{n}\right)$, exists that is best for all (or several equivalent ones); in addition there exists such a number $P$, that

$$
\begin{aligned}
\bar{x}_{i} & \geqslant 0, \lambda_{i} f_{i}\left(a_{i}-\bar{x}_{i}\right)=\bar{w}, i=1, \ldots, p \\
\bar{x}_{i} & =0, \quad \lambda_{i} f_{i}\left(a_{i}\right)<\bar{w}, \quad i=p+1, \ldots, n, \\
\text { where } \quad \bar{w}^{\top} & =f^{0}\left(\bar{x}_{1}, \ldots, \bar{x}_{p} ; x_{p+1}=\ldots=x_{n}=0\right) .
\end{aligned}
$$

Players with the numbers $p+f, \ldots, n$ may be called "egoists" since they knowingly do not set aside means for social interests. Iet us note, that in comparison with non-egoistic players, the egoism of a given player is a result of the small value $\lambda_{i} f_{i}\left(a_{i}\right)$ for himself (low opportunities or high personal pretensions). Therefore, one may speak of the egoism of players only in relation to an. actual society.

The algorithm for searching for the best equilibrium situation for a two-level society consists of the following: sequentially for $p=1, \ldots, n$ (in correspondence to the ordering of players ( 8 )) a maximization problem $\bar{w}(11)$ is solved under conditions (9) and $0 \leqslant \bar{x}_{i} \leqslant a_{i}, i=1, \ldots, p \quad$. Conditions (10) are verified with the results obtained, and if they have been fulfilled $(p \leqslant n-1)$, then the given situation is an equilibrium one. If $p=n$, then the two-level society is harmonious. by the expression

It is not difficult to show, that if the weights are given

$$
d_{i}=f^{0}\left(a_{1}, \ldots, a_{n}\right) I_{f}\left(a_{i}\right), i \in \rho^{0},
$$

then the society is hamnonious. Expression (12) may be interpreted as the measuring by each player of the importance of personal and social criteria in proportions equal to the relationship of maximal possible values of these criteria.

As is well known, equilibrium situations are stable, in that sense that it is not advantageous for the player to deviate from the situation if all of the others adhere to it. It turns out that in a two-level society the best equilibrium situation is stable 
in relation to deviations from it on the part of any aggregate of players (if only they do not form coalitions with side payments). During such a deviation the results decrease at least for ane player of the given aggregate. This property may be referred to as strong stability. In particular, it follows from this that the set of the best equilibrium situations are the Pareto set.

\section{Iinear components.}

As an example, let us consider a two-level society with linear components of the criteria vector

$$
w_{i}=\min \left\{\sum_{s=1}^{n} c_{s} x_{s}, d_{i}\left(a_{i}-x_{i}\right)\right\}, i \in \rho^{2}(13)
$$

where the number of players are ordered in accordance with (8).

Using theorem 2, we obtain that in an equilibrium situation

$$
\begin{array}{ll}
x_{i}=a_{i}-\frac{1}{\lambda_{i}} \sum_{s=1}^{p} c_{j} a_{1} /\left(1+\sum_{s=1}^{p} \frac{c_{j}}{\lambda_{s}}\right), & i=1, \ldots, p \\
x_{i}=0, & i=p+1, \ldots, n .
\end{array}
$$

Players with numbers $p+1, \ldots, n$ are egoists. It may be shown that in order that the society (13) in an equilibrium situation be harmonious, the fulfilment of the following conditions are necessary and sufficient

$$
d_{n} a_{n}>\sum_{s=1}^{n-1} c_{s} a_{s} /\left(1+\sum_{s=1}^{n-1} \frac{c_{s}}{k_{1}}\right)
$$

\section{References}

1. Iu. B. Germeier, Introduction to the Theory of Operations Research,

2. J. F. Nash, "Non-cooperative Games", Ann. Math., 1951, 54.

3. Yu. B. Germeier, I. A. Vatel, Games with a Hierarchical Vector of Interests, Communications of the Academy of Sciences of the USSR, Series on "Technical Cybernetics", No. 3, 1974 (in Russian). 